\title{
DÜBLIN
}

Technological University Dublin

ARROW@TU Dublin

\section{Horseradish and Soybean Peroxidases: Comparable Tools for Alternative Niches?}

\author{
Barry Ryan \\ Technological University Dublin, barry.ryan@tudublin.ie \\ Ciarán Fagan \\ Dublin City University, ciaran.fagan@dcu.ie \\ Neil Carolan
}

Follow this and additional works at: https://arrow.tudublin.ie/schfsehart

Part of the Life Sciences Commons

\section{Recommended Citation}

Ryan, B.J, Carolan, N. and O'Fágáin, C. (2006). Horseradish and soybean peroxidases: comparable tools for alternative niches? Trends in Biotechnology, 24, (8), 355-363. http://dx.doi.org/10.1016/ j.tibtech.2006.06.007

This Article is brought to you for free and open access by the School of Food Science and Environmental Health at ARROW@TU Dublin. It has been accepted for inclusion in Articles by an authorized administrator of ARROW@TU Dublin. For more information, please contact arrow.admin@tudublin.ie, aisling.coyne@tudublin.ie, gerard.connolly@tudublin.ie.

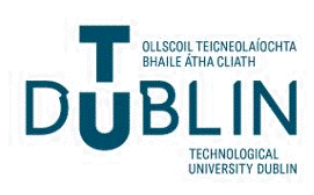


4 Barry J. Ryan, Neil Carolan and Ciarán Ó'Fágáin*.

5 School of Biotechnology and National Centre for Sensors Research, Dublin City

6 University, Dublin 9, Ireland.

Horseradish and soybean peroxidases (HRP and SBP, respectively) are useful

10 biotechnological tools. HRP is often termed the classical plant heme peroxidase, and

11 although it has been studied for decades our understanding has deepened since its cloning and subsequent expression, which has enabled numerous mutational and protein engineering studies. SBP, however, has been neglected until recently; despite offering a real alternative to HRP that actually outperforms it in terms of stability. SBP is now used in numerous biotechnological applications, including biosensors. Review of both is timely. This article summarises and discusses the main insights into the structure and mechanism of HRP, with special emphasis on HRP mutagenesis, and outlines its use in a variety of applications. It also reviews current knowledge and applications to date of SBP, particularly biosensors. The final paragraphs speculate on the future of plant heme-based peroxidases, with probable trends outlined and explored.

Key Words: Horseradish Peroxidase, Soybean Peroxidase, Review, Biosensors, Biocatalysis, Mutagenesis.

[http://www.dcu.ie/biotechnology/index.shtml]

[http://www.dcu.ie/ ncsr/]

* corresponding author: Ciarán Ó'Fágáin, (Ciaran.Fagan@dcu.ie).

Phone: +353-1-700 5288

Fax: $\quad+353-1-7005412$

Abbreviations:

ABTS, 2,2'-Azino-di-(3-ethyl-benzthiazoline-6-sulphonic acid); Co(bpy), Tris-2,2'-dipyridylocbalt(III) perchlorate trihydrate; CV, Cyclic voltammogram. DAB, 3,3'-diaminobenzidine tetrahydrochloride. DMPC, Dimyristoylphosphatidylcholine epoxidised olefins. DTT, dithiothreitol; ELISA, Enzyme-Linked Immunosorbent Assay. GOx, Glucose oxidase; GP, Graphite Powder; $\mathbf{H}_{2} \mathbf{O}_{2}$, Hydrogen peroxide; HQ, hydroquinone; IPTG, iso-propyl- $\beta$-thiogalactoside; MET, mediated electron transfer; mm, millimetre. PEGDGE, Poly(ethylene glycol) (400) diglycidylether. PelB, pectate lyase B fragment. PDB, Protein Data Bank; POs-EA, Os(bpy $)_{2} \mathrm{Cl}^{+/ 2+}$ poly(4-vinylpyridine) quaternerised with 2-bromoethylamine. Pt, Platinum. PVA, Polyvinyl alcohol. PVP, Polyvinyl pyridine. PVP-Os ${ }^{\mathbf{n}+}$, polyvinyl pyridine-osmium complex. POCT, point-of-care testing; Px, peroxidase. $\mathbf{P x}_{\mathbf{0 x}}$, oxidised peroxidase. rHRP, recombinant HRP; TTCA, poly-5,2':5',2"-terthiophene-3'-carboxylic acid. 
41 Introduction.

42 Peroxidase enzymes span the bioscience and biotechnology spectra, ranging from bioremediation [1] and biocatalysis [2] through diagnostics [3] and biosensors [4] to recombinant protein expression [5], transgenics [6], bioinformatics [7], protein engineering [8] and even to therapeutics [9]. This article contrasts two key hemecontaining plant peroxidases, horseradish (HRP) and soybean (SBP), with special emphasis on mutagenesis studies and biosensor applications. HRP is a 'traditional' enzyme, whereas SBP emerged in the 1990s. A comparison of these key biotechnological tools is timely.

\section{Brief biochemistry of horseradish and soybean peroxidase:}

52 All heme peroxidases (E.C. 1.11.1.7) have a ferriprotoporphyrin IX prosthetic group 53 located at the active site [10]. Both HRP and SBP are classified as Class III Classical 54 Secretory plant peroxidases [ $7 \& 11]$ and as such share common features (Table 1). 55 Their catalytic mechanism involves a two-electron oxidation of the heme moiety to an 56 intermediate known as Compound I. Successive one-electron reductions return the 57 enzyme to its resting state via a second intermediate, Compound II [6].

58 Determining the in vivo function of peroxidases is complex owing to the numbers of 59 isoenzymes in[E1] the family [12]. Interestingly, despite the several in vitro uses of 60 HRP, its actual in vivo role has never been elucidated. Several suggestions have been 61 proposed based on the known roles of other plant peroxidases. Peroxidases are usually 62 found in the cell wall, vacuoles, transport organelles and the rough endoplasmic 63 reticulum, and have noted roles in lignification, wound healing and auxin catabolism 64 [4]. SBP has been isolated from that plant's seed coat and its presence prevents 65 premature germination[E2] [13]. Plant peroxidases can use lignin and other plant 66 compounds as reducing substrates. Indeed, SBP has been noted to polymerise 67 coniferyl alcohol, indicating that it can efficiently catalyse reactions involving lignin 68 precursors [14]. Therefore, it is possible that peroxidases are involved in the lignification or suberisation processes of plants [15]. 
Recombinant Peroxidase Expression.

71 Recombinant hemoprotein expression has been plagued by inclusion body formation, most notably in recombinant HRP expression. Several general methods have been suggested to reduce the formation of inclusion bodies, including reducing cultivation temperature and altering inducer composition and concentration [16]. Other, more peroxidase-specific, methodologies have been cited, including use of specific E. coli strains [17], inclusion of chaperones [18] and use of leader sequences [19]. Another major obstacle in the recombinant expression of hemoproteins is the limited availabilty of heme and iron within a bacterial cell. Bacterial cells each contain $10^{5}$ to $10^{6}$ iron ions, which are essential for many metabolic pathways [20]. Culture supplementation with the heme precursor $\delta$-aminolevulinic acid has been suggested [21]; but Goodwin and co-workers have recently developed an elegant co-expression system incorporating a membrane heme receptor, allowing the use of exogenous heme as an iron source [22]. Jung and co-workers [5] noted an increased ratio of holoprotein to apoprotein with less-intense induction conditions, suggesting that slow recombinant hemoprotein production appears to allow easier incorporation of the available heme into the apoprotein [5]. Developments in HRP expression in both prokaryotic and eukaryotic systems are outlined in the supplementary online material.

\section{HRP: Cloning and Expression.}

The gene coding for the HRP protein was first synthesised by Smith and co-workers [23] based on the protein sequence published by Welinder [24]. This 940 basepairs synthetic gene was designed using commonly used codons in E. coli to minimise protein truncation owing to codon bias [25]. Recombinant HRP was over-expressed by induction but this led to the formation of misfolded apoprotein and the requirement to disrupt these aggregates, refold the protein correctly and add the heme centre. Disruption involved addition of EDTA to chelate ions, lysozyme and DNAse to reduce viscosity of the bacterial cell lysate, urea to solubilise the protein, and dithiothreitol (DTT) to break disulphide bonds. Refolding required slow exchange of disrupting reagents with folding facilitators such as calcium (for structural integrity), oxidized glutathione (to reform disulphide bridges) and hemin (to provide the prosthetic heme group) [23]. 


\section{SBP: Cloning and Expression.}

104 The gene encoding SBP was first derived from a soybean plant cDNA library 105 screened with a peroxidase-specific probe [26]. The open reading frame for the SBP

106 protein was cloned into the pET-34b (+) expression vector; however, induction of 107 rSBP led to inclusion body formation and E. coli cell death. Active SBP was achieved 108 by a refolding strategy similar to that of HRP. Unlike rHRP refolding, however, 109 inclusion of oxidised glutathione in the refolding medium decreased active SBP 110 recovery, possibly due to the formation of mispaired disulphides [26]. Henriksen and 111 co-workers [15] also developed a recombinant SBP for crystallisation studies based 112 on previous cDNA work, in which they successfully refolded SBP from inclusion 113 bodies using a cocktail that included both oxidized and reduced glutathione [27].

114 There have been several other recent examples of recombinantly expressed plant 115 peroxidases including Hushpulian and co-workers' [28] work on tobacco anionic 116 peroxidase. 
117 Mutagenesis of HRP.

118 Mutagenic studies on HRP began following successful cloning of a synthetic HRP 119 gene [23]. Before the elucidation of the crystal structure [29], most mutations focused 120 on ascertaining the key residues in the active site. Before 1997, researchers based their 121 assumptions on crystal structures of closely related peroxidases, such as cytochrome $c$ 122 peroxidase, which suggested positions 38 through 42 as key catalytic residues. 123 Mutations of Arg 38, Phe 41 and His 42 led to dramatic decreases in peroxidase 124 catalytic activity. However, some Phe 41 mutants revealed an augmented thioether 125 sulfoxidation activity owing to increased access channel area [30].

126 Asn 70 was also noted as an important residue in HRP catalysis: although it lies some 127 distance from the heme iron atom, it is hydrogen-bonded to the side chain of the distal 128 His 42. [31] Mutations in this region showed a decrease in HRP activity and a re129 orientation of active site residues. Mutation of Phe 221 altered the heme iron of the 130 resting enzyme to a quantum mixed-spin state [32]. Substitutions of Trp 117 revealed 131 this residue's role in protein folding and electron transfer [33]. Mutations within the 132 active site entrance revealed the key role of Phe 142 in binding aromatic molecules 133 [34], whilst mutations within the proximal region (the area below the heme plane) 134 disclosed the parts played by Phe 179 in aromatic molecule binding [35] and by His 135170 in heme group anchorage [36]. Table 2 lists the various site directed HRP 136 mutants. Recent examples of site directed mutants of HRP have been fewer [37], but 137 Colas and de Montellano [38] identified the key role of carboxylate side chain amino 138 acids in HRP protein-heme interactions.

139 To date, there have been few reports of HRP random mutagenesis. Arnold and co-

140 workers directionally evolved HRP with the aim of increasing activity and stability.

141 Development of a stabilised recombinant HRP is of great importance to increase and 142 consolidate the range of peroxidase applications. Three rounds of random mutagenesis 143 improved expression in yeast, yielding a nine-position HRP mutant displaying an 85-

144 fold increase in activity over the parental molecule. One round of random mutagenesis 145 was also carried out to improve stability, resulting in three mutants more stable than 146 the parent in relation to temperature and $\mathrm{H}_{2} \mathrm{O}_{2}$ tolerance (supplementary online 147 material) [8, 19, 39, \& 40]. Recent publications suggest that targeted, "semi-rational", 148 evolution of enzymes might yield superior mutants in less time [41 and references 149 within]. Mendive and co-workers developed a rapid screening methodology for 
150 random mutants displaying increased peroxidase activity, using DAB as substrate.

151 Whereas Arnold and co-workers expressed HRP in E. coli, S. cerevisiae. and $P$.

152 pastoris, Mendive and co-workers utilised a baculovirus expression system [42].

153 No mutagenesis studies on recombinant SBP have been reported to date, and the

154 authors believe that this requires urgent attention. For example, mutagenesis could

155 reveal which residues in SBP endow it with its enhanced stability vis a vis HRP [15].

156 Also, similar to HRP, SBP could be subjected to focussed directed evolution to

157 increase the number of substrates accepted.

158

\section{HRP in Biosensors}

160 One of the most common uses of HRP is in biosensors. A biosensor is "an analytical 161 device that brings together an immobilised biological sensing material [often HRP] 162 and a transducer to produce an electronic signal that is proportional to the 163 concentration of the target chemical substance" [43]. Although reports of SBP-based 164 biosensors are emerging, HRP biosensor research dominates and has continued to 165 develop through many forms, from the traditional voltammetric- and amperometric166 based methods of detection, to nano-sized devices. Real time quantification of 167 hydrogen peroxide continues to be one of the main reasons for sensor development 168 [44], although other diverse applications include the detection of glucose [45], ethanol 169 [46] and tumour markers in vivo [47]. Enzyme-based biosensors require rapid and 170 uniform transfer of electrons generated at the enzyme active site to the transducer.

171 The distance between the active site and the transducer can hinder electron transfer;

172 often, posttranslational modifications such as glycosylation increase this distance.

173 Recombinant HRP, devoid of glycans, offers a shorter path for electron transfer and 174 numerous reports of rHRP-based sensors have appeared [48 and references 175 within].While a detailed review of HRP-based sensors is beyond the scope of this 176 article, we outline some of the emerging trends in HRP-based biosensor development. 177 Biosensors, including immunosensnors [49] and electrosensors [50], incorporating 178 organic solvents have developed as an expanding area of peroxidase research, 179 primarily due to insolubility of many analytes in aqueous solutions. Recently, Konash 180 and Magner [51] developed a HRP-immobilised, mediated $\mathrm{H}_{2} \mathrm{O}_{2}$ sensor, which 181 demonstrated good catalytic activity in 2-butanone and ethyl acetate. Organic solvent 182 compatible bi-enzyme peroxidase sensors have also been cited in the literature [52]. 
183 Size reduction remains a pivotal area in sensor research. The use of nanoparticles

184 offers increased surface area for enzyme immobilisation, whilst simultaneously 185 reducing apparatus size [53]. Currently, HRP-based nano-sensors are at the forefront 186 of biosensor research [54 and Table 3].

\section{SBP in biosensors.}

189 Although HRP is the classical heme peroxidase, there is increasing interest in SBP.

190 SBP has advantages over HRP in terms of catalytic activity and stability [55]; these

191 can be exploited in biosensors. Also, unlike HRP, SBP is active in the pH range 2-6, 192 offering a greater range of potential biosensing applications [56]. The first SBP 193 biosensor was reported in 1995 by Vreeke and co-workers [57] as a thermostable 194 wired enzyme electrode using an osmium-based mediator, which aids electron transfer 195 from the active site to the electrode, modified by an epoxide. Kenausis and co196 workers [58] also used a poly(4-vinylpyridine) polymer, complexing the pyridine 197 nitrogens to the osmium-based mediator, quaternised with 2-bromoethylamine. Until 198 the use of SBP by Heller and Vreeke [59], no peroxidase-based sensors could be used 199 at $37^{\circ} \mathrm{C}$ for an extended period ( 100 hours). Monitoring of glucose "in vivo" for 200 diabetes mellitus, and of lactate for confirmation of hypoxia and ischemia, are vital in 201 patient management; use of thermostable SBP immobilised into a mediator enables 202 this [59].

203 The typical electrochemical reactions of a $\mathrm{H}_{2} \mathrm{O}_{2}$ sensing, peroxidase based, osmium204 mediated electrode system are outlined in Box 1 [60]. In addition to conveying 205 electrons, generated at the active site, to the electrode surface, the pyridin-N-ethylene 206 groups of the osmium-containing mediator also increase the hydration and provide 207 primary amines for cross-linking [58].

208 Table 3 summarizes reports to date of SBP-based biosensors, most of which use a 209 mediator. $\mathrm{H}_{2} \mathrm{O}_{2}$ can be electrochemically detected by its electrooxidation on a $\mathrm{Pt}$ (or 210 other inert Pt group metal) electrode [59]. Utilisation of an enzyme/mediator system 211 produces a multi-step mediated electron-transfer (MET) process, in which each step 212 transports the electron a small distance [61]. However, use of a mediator with a redox 213 enzyme can create its own problems: the mediator-enzyme film can, depending upon 214 its thickness, obstruct substrate diffusion [62]. Chemical modification of redox 215 enzymes with an electron relay moiety can increase multi-step MET by decreasing the 
216 electron transfer distance. This leads to improved electrical communication between

217 the enzyme's redox centre and its external environment [61].

\section{Peroxidase based Micro- and Nano-Systems}

220 An emerging field in peroxidase research is the use of micro- and nano- sized 221 structures in diagnostic and biosensing fields. Enzyme microreactors, for example, 222 permit chemical and biochemical reactions to be carried out on a microscale. HRP has 223 previously been used as a model microreactor system to monitor HRP catalytic 224 activity and as a diagnostic tool; however, the development of this field will be 225 determined by the ability to immobilise peroxidases onto suitable support structures 226 [63]. Miniaturisation of enzymatic processes is also evident in the diagnostics sector, 227 in "Lab on a Chip" and "Point of Care Testing"(POCT) research. The classical 228 application of HRP in POCT is Clinistix ${ }^{\mathrm{TM}}$; however, recently Cho and co-workers 229 [64] have applied HRP to a portable sequential cross flow immunoanalytical device. 230 Cross flow imumunoassays are capable of introducing the antigen-antibody complex 231 to the flow cell whilst sequentially extracting the catalytic signal, thus simplifying the 232 complex traditional ELISA procedure. This device demonstrates many advantages 233 over ELISA-based analytical methodologies including a rapid, sensitive and 234 inexpensive in-situ diagnosis for the presence of Hepatitis B surface antigen in a 235 sample. Miniaturisation of peroxidase-based devices also features in other research 236 fields, including the use of micro-crystals for oxidoreductase-based catalysis in 237 organic solvent [65], nano-immobilisation techniques for peroxidase based 238 wastewater treatment [66], and a more widespread use of nano-structures for 239 peroxidase based sensors [67 \& 68]. Recently Yan and co-workers [69] described a 240 microcantilever based biosensor, modified with $\mathrm{HRP}$, for $\mathrm{H}_{2} \mathrm{O}_{2}$ detection. In this 241 system the enzyme-functionalised microcantilievers deflected irreversibly in response 242 to $\mathrm{H}_{2} \mathrm{O}_{2}$ concentrations in the nanomolar range. The deflection was caused by 243 conformational change within the HRP molecule as it underwent oxidation by the $244 \mathrm{H}_{2} \mathrm{O}_{2}$; the irreversibility was due to the absence of a second, reducing substrate 245 required for reversion of HRP to the resting state. This technique may also provide a 246 sensitive tool for investigating protein structural change. HRP has also been utilised as 247 the functional component of self-assembled three-dimensional (3-D) nano-structures. 248 Rauf and co-workers [70] utilised self-assembly layer-by-layer technology to 
construct controlled 3-D catalytically active nano-structures. This method of

250 peroxidase immobilisation allows for increased catalytic activity per unit area, and

251 will aid in the miniaturisation of biosensors, biochips and immobilised biocatalysts.

252 With increasing sophistication of support structures on the micro- and nano-scale,

253 miniaturisation of peroxidase-based devices will continue to develop in the future,

254 particularly in the fields of POCT and biosensing.

255

256 Peroxidase based Biocatalysis.

257 A major shortcoming of all heme-dependent peroxidases is their low operational 258 stability, owing to oxidative degeneration of the heme group [71]. Operational 259 stability of SBP can be increased by generating $\mathrm{H}_{2} \mathrm{O}_{2}$ in situ from glucose and $\mathrm{O}_{2}$.

260 When co-immobilized with glucose oxidase in a polyurethane foam, SBP could act as 261 a peroxygenase to convert thioanisole to its sulphoxide (i.e. by inserting an oxygen 262 atom). Here, SBP uses the $\mathrm{H}_{2} \mathrm{O}_{2}$ generated in situ by glucose oxidase; it formed no 263 sulphoxide with free, exogenous $\mathrm{H}_{2} \mathrm{O}_{2}$ [71]. Such an arrangement avoids excessive 264 initial $\mathrm{H}_{2} \mathrm{O}_{2}$ concentrations and, hence, formation of compound III (a reversible dead265 end complex formed from compound II in the presence of an excess $\mathrm{H}_{2} \mathrm{O}_{2}$, which 266 slowly reverts to native enzyme; [10]) and/or irreversibly inhibited SBP. HRP has 267 been subjected to intense experimentation, including a large body of work focussing 268 on site directed mutants (see above and Table 2). Now that the key catalytic residues 269 are known, researchers have begun to use site directed mutagenesis to alter the 270 function of the HRP molecule [48], e.g. by construction of an improved luminol 271 binding site [72]. Directed evolution stabilised HRP against thermal denaturation [39] 272 and has endowed it with increased $\mathrm{H}_{2} \mathrm{O}_{2}$ tolerance and increased catalytic activity 273 [40]. Further targeted directed evolution, focussing on the substrate access channel 274 and binding pocket, could allow HRP to accept an increased variety of substrates [73], 275 and promote further diversification of HRP applications in organic synthesis [41]. 276 Peroxidase catalysis in organic solvents, both aqueous and anhydrous, offers a huge 277 advantage to organic chemists, as difficult asymmetric oxidation and reduction 278 reactions can take place rapidly and with high specificity [74]. The major problems of 279 substrate solubility and unwanted side reactions promoted by water are also overcome 280 during organic solvent based synthesis. Additionally, in some anhydrous solvents 281 peroxidase (HRP and SBP) activity was actually increased [75], with additional 
282 methods, such as salt activation [76] and excipient aided lyophilisation [77] also

283 resulting in increased peroxidase activity. However, in some low water solvents, 284 peroxidases can lose their confirmational structure [78]; although recent advances in 285 peroxidase encapsulation in amphiphilic matrices [79], the use of reverse micelles 286 [80] and oil emulsions [81] allow for peroxidase activity in an extended range of 287 anhydrous solvents. Reactions carried out in these solvents include hydroxylations, N288 demethylations and sulphoxidations [2]. An interesting recent environmental 289 application of SBP polymerisation in organic solvents is the production of 290 polycardanol in as a potential anti-biofouling agent [82], whilst recently it has been 291 noted that HRP requires a mediator to catalyse the same substrate [83]. The interested 292 reader is directed to a recent review of this area [74].

\section{Peroxidase based Bioremediation.}

295 Highly expressed, stabilized, recombinant HRP [39, 40] could be very useful for 296 wastewater cleanup, provided the recombinant enzyme can be produced cost 297 effectively and in sufficient quantities. Phenol cleanup by HRP has been widely 298 reviewed [1] but several drawbacks limit its widespread application, including 299 intolerance of high concentrations of the primary substrate $\mathrm{H}_{2} \mathrm{O}_{2}$ [84], low enzymatic 300 reusability, and financial costs. Plant heme peroxidase expression in E.coli can be 301 frustrating, but advances in peroxidase expression, without formation of inclusion 302 bodies, may pave the way for increased production of recombinant peroxidases (See 303 supplementary online material). SBP has proven itself a worthy alternative 304 peroxidase: it displays superior stability and activity characteristics to the classical 305 HRP. However, research into this enzyme lags far behind HRP. SBP can effectively 306 cleanup phenolic wastewater, yet recent publications cite HRP as being a superior, 307 albeit less stable, catalyst than SBP for phenol cleanup [85]. Development of an 308 enhanced catalytic SBP mutant would provide a powerful tool for wastewater 309 treatment. Bódalo and co-workers [85] noted that the choice of peroxidase for 310 wastewater treatment also depends on effluent characteristics, operational 311 requirements and costs. SBP has been shown to outperform HRP in oxidative dye 312 removal [86]. SBP, possibly owing to its larger substrate access channel, and, hence, 313 greater exposure of the catalytically important delta heme edge, can accept more 314 substrates than HRP [87]. 


\section{HRP: an Unlikely Therapeutic}

317 An exciting application of HRP is as a novel cancer treatment via gene-directed 318 enzyme/prodrug therapy. It has been noted that the non-toxic HRP substrate, indole-3319 acetic acid (IAA), forms a radical that is toxic to cancer cells upon HRP catalysis. The 320 exact mechanism of toxicity remains unclear: it is believed to involve lipid 321 peroxidation induced by the free radical formation [88]; however, in human 322 melanoma cells, death receptor-mediated and mitochondrial apoptotic pathways are 323 known to be involved also [89]. Leaving aside the actual reason(s) for toxicity, 324 inactive IAA can be introduced to the body; and then becomes activated by HRP at 325 the region of interest. Localisation of the HRP molecule is achieved via its 326 conjugation to an antibody specific to an extracellular tumour antigen. This approach 327 has become the focus of much research and numerous clinical trials, due to several 328 attractive features: these include the robust nature of the activating enzyme and the 329 low toxicity of the prodrug [90]. HRP has been shown to activate other pro-drugs 330 including ellipticine [91] and halogenated IAA derivates [92]. The interested reader is 331 directed to the excellent recent review of Dachs et al. [93]. 
333 As outlined, peroxidases are widely studied and very important enzymes, with many 334 applications in the life sciences and beyond. They remain pivotal to advancing 335 biotechnology, and as such, we present two clearly distinct, yet similar members of 336 this classical family. Continued research into the "traditional" HRP, has been 337 accompanied by the slow, but steady progression of SBP. Crude SBP, isolated from 338 waste soybean hulls, offers a cheap bulk peroxidase catalyst for applications such as 339 wastewater treatment and organic synthesis, whilst the more costly peroxidase 340 alternatives (plant HRP and recombinant HRP and SBP) will prove themselves in 341 higher value niches, such as diagnostics and therapeutics. With improved 342 understanding of the catalytic and stability characteristics, the detection of new 343 substrates and the increasing use of implantable devices in the medical field, SBP will 344 rapidly develop its own high value market niche. As noted for HRP, use of 345 recombinant SBP would also benefit the biosensor field by permitting more rapid 346 electron transfer, due to the lack of protein glycosylation. Improvement of these two 347 peroxidases, by rational mutation and "focussed" directed evolution, will widen their 348 applications and expand their roles as key biotechnological tools in the future.

We thank the following for financial support: the Irish Research Council for Science, Engineering and Technology (Embark Initiative postgraduate scholarship, BJR) the National Centre for Sensors Research and School of Biotechnology, Dublin City University (NC) and the Research Advisory Panel of Dublin City University (Albert College Award, C.Ó'F.) (The National Centre for Sensors Research was established under the Programme for Research in Third Level Institutions of the Higher Education Authority) 
1. Wanger M. and Nicell J.A. Detoxification of phenolic solutions with horseradish peroxidase and hydrogen peroxide. (2002), Water Research, 36, (16), 4041-4052.

2. van de Velde F. et al. (2001), Improving the catalytic performance of peroxidase in organic synthesis. Trends in Biotechnology, 19, (2), 73-80.

3. Dotsikas Y. and Loukas Y.L. (2004). Employment of 4-(1-imidazolyl)phenol as a luminol signal enhancer in a competitive-type chemiluminescence immunoassay and its comparison with the conventional antigen-horseradish peroxidase conjugate-based assay. Analytica Chimica Acta, 509, (1), 103-109.

4. Azevedo A.M. et al. (2003). Horseradish peroxidase: a valuable tool in biotechnology. Biotechnology Annual Review, 9, 199-247.

5. Jung Y. et al. (2001). High-level production of heme-containing holoproteins in Escherichia coli. Applied Microbiological Biotechnology, 55, (2), 187-191.

6. Veitch N.C. (2004). Horseradish peroxidase: a modern view of a classic enzyme. Phytochemistry, 65, (3), 249-259.

7. Duroux L. and Welinder K.G. (2003). The Peroxidase Gene Family in Plants: A Phylogenetic Overview. Journal of Molecular Evolution, 57, (4), 397-407.

8. Morawski B. et al. (2000). Functional expression of horseradish peroxidase in Saccharomyces cerevisiae and Pichia pastoris. Protein Engineering, 13, (5), 377-384.

9. Tupper J. et al. (2004). Use of horseradish peroxidase for gene-directed enzyme prodrug therapy with paracetamol. British Journal of Cancer, 90, (9), 1858-1862.

10. Dunford HB. (1999). "Heme Peroxidases". Wiley and Sons, New York, pp 1-111[E3].

11. Welinder K.G. (1992). Superfamily of Plant, fungal and bacterial peroxidases. Current Opinion in Structural Biology, 2, 388-393.

12. Hoyle M.C. (1977). High-resolution of peroxidase-indoleacetic acid oxidase isoenzymes from Horseradish by isoelectric-focusing. Plant Physiology, 60, 787-793.

13. dos Santos W.D. et al. (2004). Lignification and related enzymes in Glycine max root growthinhibition by ferulic acid. Journal of Chemical Ecology, 30, (6), 1203-1212.

14. Schmitz et al, (1997). Characterisation of anionic Soybean Peroxidase (Glycine Max) Seed coat peroxidase. Canadian Journal of Botany, 75, 1336-1341.

15. Henriksen A. et al. (2001). Structure of soybean seed coat peroxidase: A plant peroxidase with unusual stability and haem-apoprotein interactions. Protein Science, 10, (1), 108-115.

16. Hoffmann F. et al. (2004). Minimising inclusion body formation during recombinant protein production in Escherichia coli at bench and pilot scale. Enzyme and Microbial Technology, 34, (34), 235-241.

17. Teilum K. et al. (1999). Disulphide bond formation and folding of plant peroxidases expressed as inclusion body protein in Escherichia coli thioredoxin reductase negative strains. Protein Expression and Purification, 15, (1), 77-82.

18. Zhang Y. et al. (1998). Expression of eurkaryotic proteins in soluble form in Escherichia coli. Protein Expression and Purification, 12, (2), 159-165.

19. Lin Z. et al. (1999). Functional Expression of Horseradish Peroxidase in E. coli by directed evolution. Biotechnology Progress, 15, 467-471.

20. Wandersman C. and Stojiljkovic I. (2000). Bacterial heme sources: the role of heme, hemoprotein receptors and hemophores. Current Opinion in Microbiology, 3, (2), 215-220.

21. Woodard S.I. and Dailey H.A. (1995). Regulation of Heme Biosynthesis in Escherichia coli. Archives of Biochemistry and Biophysics, 316, (1), 110-115

22. Varnado C.L and Goodwin D.C. (2004). System for the expression of recombinant hemoproteins in Escherichia coli. Protein Expression and Purification, 35, (1), 76-83.

23. Smith A.T. et al. (1990). Expression of a synthetic gene for horseradish peroxidase in Escherichia coli and folding and activation of the recombinant enzyme with $\mathrm{Ca}^{2+}$ and Heme. Journal of Biological Chemistry, 265, (22), 13335-13343.

24. Welinder K G. (1979). Amino acid sequence studies of horseradish peroxidase. Amino and carboxy termini, cyanogen bromide and tryptic fragments, the complete sequence, and some structural characteristics of horseradish peroxidase C. European Journal Biochemistry, 96, 483502.

25. Gustafsson C. et al. (2004). Codon Bias and heterologous protein expression. Trends in Biotechnology, 22, (7), 346-353.

26. Chen H. and Vierling R.A. (2000). Molecular cloning and characterisation of soybean peroxidase gene families. Plant Science, 150, (2), 129-137.

27. Gijzen M. (1997). A deletion mutation at the ep locus causes low seed coat peroxidase activity in soybean. The Plant Journal, 12, (5), 991-998. 
28. Hushpulian D.M. (2003). Expression and refolding of tobacco anionic peroxidase from E-coli inclusion bodies. Biochemistry (Moscow), 68, (11), 1189-1194.

29. Gajhede M. et al. (1997). Crystal structure of horseradish peroxidase C at $2.15 \AA$ resolution. Nature Structural Biology, 4, (12), 1032-1038.

30. Ozaki S. and Ortiz de Montellano P.R. (1995). Molecular engineering of horseradish peroxidase. Thioether sulfoxidation and styrene epoxidation by Phe-41 leucine and threonine mutants. J.Am.Chem.Soc., 117, (27), 7056-7064.

31. Nagano S, Tanaka M, Ishimori K, Watanabe Y. and Morishima I. (1996). Catalytic roles of the distal site asparagine-histidine couple in peroxidases. Biochemistry, 35, (45), 14251-14258.

32. Howes B.D et al. (2001). Heme-linked interactions in horseradish peroxidase revealed by spectroscopic analysis of the Phe-221 to Met mutant. Biochem. J., 353, (2), 181-191.

33. Ignatenko O.V. et al. (2000). Catalytic properties of tryptophanless recombinant horseradish peroxidase. Biochemistry (Moscow), 65, (5), 583-587.

34. Veitch N.C. et al. (1995). Solution characterisation by NMR spectroscopy of two horseradish peroxidase isoenzyme $\mathrm{C}$ mutants with alanine replacing either Phe142 or Phe143. European Journal of Biochemistry, 233, (2), 650-658.

35. Veitch N.C. et al. (1997). Identification of a critical phenylalanine residue in horseradish peroxidase, Phe179, by site-directed mutagenesis and H-NMR. Implications for complex formation with aromatic donor molecules. Biochemistry, 36, (48), 14751-14761.

36. Newmyer S.L. et al. (1996). Rescue of the horseradish peroxidase His-170 to Ala mutant activity by imidazole. Importance of proximal ligand tethering. Biochemistry, 35, (39), 12788-12795.

37. Howes B.D. et al. (2005). Spectroscopic and kinetic properties of the horseradish peroxidase mutant T171S. FEBS Journal, 272, 5514-5521

38. Colas C. and de Montellano P. R. O. (2004). Horseradish Peroxidase Mutants That Autocatalytically Modify Their Prosthetic Heme Group. Journal of Biological Chemistry, 279, (23), 24131-24140.

39. Arnold F.H. and Lin, Z. (2000). California Institute Of Technology "Expression of Functional Eukaryotic Proteins". Worldwide Patent: PCT/US99/17127, WO 00/006718.

40. Morawski B. et al. (2001). Functional expression and stabilisation of HRP by directed evolution in S. cerevisiae. Biotechnology and Bioengineering, 76, (2), 99-107.

41. Kazlauskas R. (2005). Biological chemistry: Enzymes in focus. Nature, 436, 1096-1097.

42. Mendive F.M. et al. (2003). Construction of a mutant library of horseradish peroxidase gene by directed evolution and development of an in situ screening method. Braz. J. Chem. Eng., 20, (1), 33-38.

43. Luong J.H.T. et al. (1995). Enzyme reactions in the presence of cyclodextrins: biosensors and enzyme assays. Trends in Biotechnology, 13, 457-463.

44. Tønning E. et al. (2005). Chemometric exploration of an amperometric biosensor array for fast determination of wastewater quality. Biosensors and Bioelectronics, 21, (4), 608-617.

45. Alonso Lomillo M. A. et al. (2005). Biosensor based on platinum chips for glucose determination. Analytica Chimica Acta, 547, (2), 209-214.

46. Azevedo A. M. et al. (2005). Ethanol biosensors based on alcohol oxidase. Biosensors and Bioelectronics, 21, (2), 235-247.

47. Lin J. and Ju, H. (2005). Electrochemical and chemiluminescent immunosensors for tumour markers. Biosensors and Bioelectronics, 20, (8), 1461-1470.

48. Ferapontova E. (2004). Direct peroxidase bioelectrocatalysis on a variety of electrode materials. Electronanalysis, 16, (13), 1101-1112.

49. Penalva J. et al. (1999). Analytical properties of Immunosensors working in organic solvents. Analytical Chemistry, 71, 3862-3872.

50. Campanella L. et al. (1999). Analysis of several real matrices using mono-, bi-enzymatic or inhibition organic phase enzyme electrodes. Analytica Chemica Acta, 393, 109-120.

51. Konash, A. and Magner E. (2005). Electrochemically Mediated Reduction of Horseradish Peroxidase by 1,1'-Ferrocene Dimethanol in Nonaqueous Solvents, Anal. Chem., 77,1647-1654.

52. Castillo J. et al. (2003). Bienzyme biosensors for glucoe, ethanol and putrescine built on oxidase and sweet potato peroxidase. Biosensors and Bioelectronics, 18, 705-714.

53. Liu, Y.-L. et al. (2005). Nanosized flower-like ZnO synthesized by a simple hydrothermal method and applied as matrix for horseradish peroxidase immobilization for electro-biosensing. Journal of Inorganic Biochemistry, 99, (10), 2046-2053.

54. Wu L et al. (2006). Electrochemical immunoassay for CA125 based on cellulose acetate stabilized antigen/colloidal gold nanoparticles membrane. Electrochimica Acta, 51, (7), 1208-1214. 
55. Kamal J.K. and Behere DV. (2003). Activity, stability and conformational flexibility of seed coat soybean peroxidase. Journal of Inorganic Biochemistry, 94, (3), 236-42.

56. Wang B.Q. et al. (1999). Sol-Gel thin film immobilized soybean peroxidase biosensor for the amperometric determination of hydrogen peroxide in acid medium. Analytical Chemistry, 71, 1935-1939.

57. Vreeke M.S. et al. (1995). A thermostable hydrogen peroxide sensor based on "wiring" of soybean peroxidase. Analytical Chemistry, 67, 4247-4249.

58. Kenausis G. et al. (1997). Electrochemical glucose and lactate sensors based on "wired" thermostable soybean peroxidase operating continuously and stably at $37^{\circ} \mathrm{C}$. Analytical Chemistry, 69, 1054-1060.

59. Heller A. and Vreeke M. S. (1997). E. Heller and Company. Soybean peroxidase electrochemical sensor. United States Patent. US. 5665222.

60. Li W. et al. (2000). Fabrication of multilayer films containing horseradish peroxidase and polycation-bearing Os complex by means of electrostatic layer-by-layer adsorption and its applications as a hydrogen peroxide sensor. Analytica Chimica Acta. 418, 225-232.

61. Katz, E. (2002). Mediated electron-transfer between redox-enzymes and electrode supports. IN. A. Bard and J. Stratmann. Encyclopaedia of Electrochemistry, Bioelectrochemistry, 9, New York. John Wiley and Sons Inc, 561-626.

62. Sun Y. et al. (1998). Chemically modified electrode via layer-by-layer deposition of glucose oxidase (GOD) and polycation-bearing Os complex. Thin Solid Films, 327-329, 730-733.

63. Verpoorte E. (2002). Microfluidic chips for clinical and forensic analysis. Electrophoresis, 23, (5), 677-712.

64. Cho J-H. et al. (2005). An enzyme immunoanalytical system based on sequential cross-flow chromatography. Analytical Chemistry, 77, 4091-4097.

65. Kreiner M. and Parker M-C. (2005). Protein-coated microcrystals for use in organic solvents: application to oxidoreductases. Biotechnology Letters, 27, 1571-1577.

66. Patel D.S. et al. (2005). Nano-assembly of manganese peroxidase and lignin peroxidase from $P$. chrysosporium for biocatalysis in aqueous and non-aqueous media. Colloids and Surfaces B, $\mathbf{4 5}$, 13-19.

67. Zhu J-J. et al. (2005). Reagentless electrochemical biosensor based on the multi-wall carbon nanotubes and nanogold particles composite film. Frontiers in Bioscience, 10, 521-529.

68. Lee Y-M. et al. (2006). Immobilisation of horseradish peroxidase on multi-wall carbon nanotubes and its electrochemical properties. Biotechnology Letters, 28, 39-43.

69. Yan X. et al. (2006). Microcantilevers modified by horseradish peroxidase: intercalated nanoassembly for hydrogen peroxide detection. Analytical Sciences, 22, 205-208.

70. Rauf S. et al. (2006). Building three-dimensional nanostructures with active enzymes by surface templated layer by layer assembly. Chem. Commun., 16, 1721-1723.

71. van de Velde F. et al. (2000) Improved operational stability of peroxidases by coimmobilization with glucose oxidase. Biotechnology and Bioengineering, 69, (3), 286-291.

72. Tanaka M. et al (1999). Luminol activity of horseradish peroxidase mutants mimicking a proposed binding site for luminol in Arthromyces ramosus peroxidase. Biochemistry, 38, (32), 10463-10473.

73. Smith A.T. and Veitch N.C. (1998). Substrate binding and catalysis in heme peroxidases. Current Opinion in Chemical Biology, 2, 269-278.

74. Klibanov A.M. (2003). Asymmetric enzymatic oxidoreductions in organic solvents. Current Opinion in Biotechnolgy, 14, 427-431.

75. Dai L. and Klibanov A.M. (2000). Peroxidase-catalysed asymmetric sulfoxidation in organic solvents versus water. Biotechnology and Bioenginerring, 70, (3), 353-357.

76. Morgan J.A. and Clark D.S. (2004). Salt activiation of nonhydrolase enzymes for use in organic solvnets. Biotechnology and Bioengineering, 85, (4), 456-459.

77. Dai L. and Klibanov A.M. (1999) Striking activation of oxidative enzymes suspended in nonaqueous media. Proc. Nat. Acad. Sci. USA, 96, 9475-9478.

78. Azevedo A.M.et al. (2001). Stability of free and immobilised peroxidase in aqueous-organic solvents mixtures. Journal Molecular Catalysis B, 15,147-153.

79. Bruns N. and Tiller J.C. (2005). Amphiphilic Network as nanoreactor for enzymes in organic solvents. Nano Letters, 5, (1), 45-48.

80. Michizoe J. et al. (2003). Control of water content by reverse micellar solutions for peroxidase catalysis in a water-immiscible organic solvent. Journal of Bioscience and Bioengineering, 95, (4), 425-427. 
81. Kamiya N. et al. (2000). Catalytic and strucutral properties of surfactant-Horseradish Peroxidase complex in organic media. Biotechnology Progress, 16, 52-58.

82. Kim Y.H. et al. (2003). Polymeriation of cardanol using soybean peroxidase and its potential application as anti-biofilm coating material. Biotechnology Letters, 25, 1521-1524.

83. Won K. et al. (2004). Horseradish peroxidase-catalysed polymerisation of cardanol in the presence of redox mediators. Biomacromolecules, 5, (1), 1-4.

84. Nicell J.A. and Wright H. (1997). A model of peroxidase activity with inhibition by hydrogen peroxide. Enzyme and Microbial Technology, 21, 302-310.

85. Bódalo A. et al. (2005). Comparison of commercial peroxidases for removing phenol from water solutions. Chemosphere, 63, (4), 626-632.

86. Knutson $\mathrm{K}$ et al. (2005). Enzymatic biobleaching of two recalcitrant paper dyes with horseradish and soybean peroxidase. Biotechnology Letters, 27, (11), 753-8.

87. Alpeeva I.S. and Sakharov I.Y. (2005). Soybean peroxidase-catalyzed oxidation of luminol by hydrogen peroxide. Journal of Agriculture and Food Chemistry, 53, (14), 5784-8.

88. Folkes L.K. and Wardman P. (2001). Oxidative activation of indole-3-acetic acids to cytotoxic species - A potential new role for plant auxins. Biochem. Pharmacol., 61, 129-136.

89. Kim D-S. et al. (2004). Oxidation of indole-3-acetic acid by horseradish peroxidase induces apoptosis in G361 human melanoma cells. Cellular Signalling, 16, 81-88.

90. Tafazoli S.and O'Brien, P.J. (2004). Prooxidant Activity and Cytotoxic effects of Indole-3-acetic Acid derivative Radicals. Chem.Res. Toxicol., 17, 1350-1355.

91. Poljaková J.et al. (2005). Oxidation of an antitumor drug ellipticine by peroxidases. Biomed. Pap. Med Fac. Univ. Palacky. Olomouc. Czech Repub., 149, (2), 449-453.

92. Rossiter S. et al. (2002). Halogenated Indole-3-acetic Acids as Oxidatively Activated Prodrugs with potential for targeted Cancer Therapy. Bioorganic and Medical Chemistry Letters, 12, 25232526.

93. Dachs G.U. et al. (2005). From bench to bedside for gene-directed enzyme prodrug therapy of cancer. Anti-Cancer Drugs. 16, (4), 349-359.

94. Welinder K.G. and Larsen Y.B. (2004). Covalent structure of soybean seed coat peroxidase. Biochimica et Biophysica Acta, 1698, (1), 121-126.

95. Wuhrer M. et al. (2005). New features of site-specific horseradish peroxidase (HRP) glycosylation uncovered by nano-LC-MS with repeated ion-isolation/fragmentation cycles. Biochimica et Biophysica Acta., 1723, (1-3), 229-239.

96. Gray J.S.S. and Montgomery R. (2006). Asymmetric glycosylation of soybean seed coat peroxidase. Carbohydrate Research, 341, (2), 198-209.

97. Paul K.G. (1963). "Peroxidases” IN The Enzymes (Boyler P.D. ed), Academic Press, 8, 227-237.

98. Rodriguez-Lopez J.N. et al. (1996). Role of Arginine 38 in horseradish peroxidase a critical residue for substrate binding and catalysis. Journal of Biological Chemistry, 271, (8), 4023-4030.

99. Newmyer S.L. and de Montellano P.R.O. (1996). Rescue of the catalytic activity of an H42A mutant of horseradish peroxidase by exogenous imidazoles. Journal of Biological Chemistry, 271, (25), 14891-14896.

100.Gazaryan I.G. et al. (1994). Effect of single-point mutations Phe-41-His and Phe-143-Glu on folding and catalytic properties of recombinant horseradish peroxidase expressed in E. coli. FEBS Letters, 354, (3), 248-250.

101. Veitch N.C. et al. (1995). Solution characterisation by NMR spectroscopy of two horseradish peroxidase isoenzyme C mutants with alanine replacing either Phe142 or Phe143. European Journal of Biochemistry, 233, (2), 650-658.

102. Howes B.D. et al. (2001). Mutation of residues critical for benzohydroxamic acid binding to horseradish peroxidase isoenzyme C. Biopolymers, 62, (5), 261-267.

103. Mukai M. et al. (1997). Effects of concerted hydrogen bonding of distal histidine on active site structures of horseradish peroxidase. Resonance Raman studies with Asn70 mutants. J.Am.Chem.Soc., 119, (7), 1758-1766.

104.Gazaryan I.G. et al. (1999) Tryptophanless recombinant horseradish peroxidase. Stability and catalytic properties. Biochem. Biophys. Res. Commun., 262, (1), 297-301.

105. Morimoto A. et al. (1998). Detection of a tryptophan radical as an intermediate species in the reaction of horseradish peroxidase mutant (Phe-221 to Trp) and hydrogen peroxide. Journal of Biological Chemistry, 273, (24), 14753-14760.

106. Wang B. et al. (2001). Acid-Stable Amperometric Soybean Peroxidase Biosensor Based on a SelfGelatinizable Grafting Copolymer of Polyvinyl Alcohol and 4-Vinylpyridine. Electroanalysis, 13, (7), 555-558. 
107.Zhang Z. et al. (2002). Direct voltammetry and catalysis with Mycobacterium tuberculosis catalase-peroxidase, peroxidases, and catalase in lipid films. Analytical Chemistry, 74, (1), 163-70.

108. Song Y. et al. (2006). A novel hydrogen peroxide sensor based on horseradish peroxidase immobilized in DNA films on a gold electrode. Sensors and Actuators B, 114, (2), 1001-1006.

109.Lei C.-X. et al. (2004). An amperometric hydrogen peroxide biosensor based on immobilizing horseradish peroxidase to a nano-Au monolayer supported by sol-gel derived carbon ceramic electrode. Bioelectrochemistry, 65, (1), 33-39.

110. Castilho J.T. et al. (2005). Amperometric biosensor based on horseradish peroxidase for biogenic amine determinations in biological samples. Journal of Pharmaceutical and Biomedical Analysis, 37, (4), 785-791.

111.Li S. and Chen A. (2005). Coadsorption of Horseradish Peroxidase with Thionine on $\mathrm{TiO}_{2}$ nanotubes for biosensing. Langmuir, 21, 8409-8413.

112. Yuan R. et al. (2005). Electrochemical characteristics of a platinum electrode modified with a matrix of polyvinyl butyral and colloidal Ag containing immobilized horseradish peroxidase. Anal. Bioanal Chem., 381, 762-768.

113. Schuvailo O.N. et al. (2005). Carbon fibre-based microbiosensors for in vivo measurements of acetylcholine and choline. Biosensors and Bioelectronics, 21, (1), 87-94.

114. Rahman M.A. et al. (2004). A performance comparison of choline biosensors: anodic or cathodic detections of $\mathrm{H}_{2} \mathrm{O}_{2}$ generated by enzyme immobilized on a conducting polymer. Biosensors and Bioelectronics, 19, (12), 1565-1571.

115. Ferapontova E. and Gorton L. (2002). Effect of pH on direct electron transfer in the system gold electrode-recombinant horseradish peroxidase. Bioelectrochemistry, 55, (1-2), 83-87.

116.Lindgren A. et al. (1999). Direct electron transfer catalysed by recombinant forms of horseradish peroxidase: insight into the mechanism. Electrochemistry Communications, 1, 171-175.

117. Shipovskov S. et al (2004). Recombinant horseradish peroxidase- and cytochrome c-based twoelectrode system for detection of superoxide radicals. Bioelectrochemistry, 63, (1-2), 277-280.

118. Ferapontova E. et al. (2001). P-Chip and P-Chip Bienzyme Electrodes Based on Recombinant Forms of Horseradish Peroxidase Immobilized on Gold Electrodes. Biochemistry (Moscow), 66, (8), 832-839. 


$\begin{array}{llll}\mathrm{H}_{2} \mathrm{O}_{2}+2 \mathrm{H}^{+}+\mathrm{Px} & \longrightarrow \mathrm{Px}_{\mathrm{ox}}+2 \mathrm{H}_{2} \mathrm{O} & \text { equation 1 } \\ \mathrm{Px}_{\mathrm{ox}}+2 \mathrm{PVP}-\mathrm{Os}^{2+} & \longrightarrow \mathrm{Px}+2 \mathrm{PVP}-\mathrm{Os}^{3+} & \text { equation 2 } \\ 2 \mathrm{PVP}-O s^{3+}+2 \mathrm{e}^{-} & \longrightarrow 2{\mathrm{PVP}-\mathrm{Os}^{2+}}^{2+} & \text { equation 3 } \\ \mathrm{H}_{2} \mathrm{O}_{2}+2 \mathrm{e}^{-}+2 \mathrm{H}^{+} & \longrightarrow \mathrm{H}_{2} \mathrm{O} & \end{array}$

Box[E4] 1: Typical biosensor based on a mediated peroxidase (Px) reaction scheme. Upon the addition of $\mathrm{H}_{2} \mathrm{O}_{2}$, Px catalyses the reaction forming water; in the process, Px goes through its catalytic cycle. This causes the mediator to go from its resting state of $\mathrm{Os}^{2+}$ to $\mathrm{Os}^{3+}$. The osmium species is seen as a one-electron donor, used as the mediator to assist in electron transfer from the active site of Px to the electrode surface. $\mathrm{Px}_{\mathrm{ox}}$ corresponds to the catalytic intermediate Compound I, formed by a two-electron oxidation. The individual one-electron reduction steps that take place on the enzyme itself (formation of catalytic intermediate Compound II and reversion to resting enzyme, Px) have been omitted from equation 2 for the sake of clarity. Adapted from Analytica Chimica Acta. 418, Li W. et al. (2000). Fabrication of multilayer films containing horseradish peroxidase and polycation-bearing Os complex by means of electrostatic layer-by-layer adsorption and its applications as a hydrogen peroxide sensor. 225-232. [60] Copyright 2000, with permission from Elsevier. 
Table 1: Comparison of the biochemical and structural properties of HRP and SBP. Key references are noted.

\begin{tabular}{|c|c|c|}
\hline & Horseradish Peroxidase & Soybean Peroxidase \\
\hline Species Name & Armoracia rusticana [23] & Glycine $\max [14]$ \\
\hline Number of Amino Acids & $306[23]$ & $306[94]$ \\
\hline Enzyme Classification & 1.11.1.7 [29] & 1.11.1.7 [15] \\
\hline PDB Accession Number & 1ATJ & $1 \mathrm{FHF}$ \\
\hline Molecular Weight & $44,100 \mathrm{Da}[6]$ & $40,660 \mathrm{Da}[15]$ \\
\hline Carbohydrate & 7,580 Da [6] & 7,400 Da [15] \\
\hline Heme Group & $550 \mathrm{Da}[6]$ & $550 \mathrm{Da}[15]$ \\
\hline Calcium Ions & $80 \mathrm{Da}[6]$ & $80 \mathrm{Da}[15]$ \\
\hline Glycosylation Sites & $\begin{array}{r}\text { Asn: } 13,57,158,186,198, \\
214,255,268,316[95]\end{array}$ & Asn: $185,197,211,216[96]$ \\
\hline pI & $9.0[24]$ & $4.1[14]$ \\
\hline pH Activity Range & $4-8[97]$ & $2-10[15]$ \\
\hline Secondary Structure & $\begin{array}{c}13 \alpha \text {-helices } \\
3 \beta \text {-sheets [29] }\end{array}$ & $\begin{array}{c}13 \alpha \text {-helices } \\
2 \beta \text {-sheets }[15]\end{array}$ \\
\hline Disulphide Bridges & $\begin{array}{c}11-91,44-49,97-301,177-209 \\
{[29]}\end{array}$ & $\begin{array}{c}11-91,44-49,97-299,176-208 \\
{[15]}\end{array}$ \\
\hline In vivo localisation & Roots, cell wall, vacuoles [6] & Hourglass cells, seed coat [14] \\
\hline
\end{tabular}


Table 2: Summary of HRP site directed mutants[E5]

\begin{tabular}{|c|c|}
\hline Mutations & Conclusions \\
\hline \multicolumn{2}{|c|}{ Active Site. $[98,99, \& 100]$} \\
\hline R38A $\quad$ F41T & Arg38 and His42 are key residues in enzyme catalysis. \\
\hline $\mathrm{F} 41 \mathrm{~V}$ & Arg 38 and His 42 mutations decrease Compound I formation. \\
\hline F41W & Mutants affect reactivity towards reducing substrates. NB- Morimoto ref. \\
\hline $\mathrm{H} 42 \mathrm{~A}$ & Arg 38 and His 42 are acid base partners. Arg 38 stabilises His 42. \\
\hline $\mathrm{H} 42 \mathrm{E}$ & Arg38 and His42 operate in concert to distally bind BHA. \\
\hline $\mathrm{H} 42 \mathrm{~L}$ & Arg38 and His42 are dioxygen-heterolytic cleavers \\
\hline H42Q & Arg38 plays a role in $\mathrm{H}_{2} \mathrm{O}_{2}$ binding and cleavage. \\
\hline $\mathrm{H} 42 \mathrm{R}$ & Hydrophobicity of active site region is critical in enzymatic catalysis. \\
\hline $\mathrm{H} 42 \mathrm{~V}$ & Space creating active site mutants alter substrate specificity. \\
\hline $\mathrm{F} 41 \mathrm{~L}$ & Phe41 acts as hydrophobic barrier between Arg38 and His 42 . \\
\hline \multicolumn{2}{|c|}{ Active Site Entrance. [101] } \\
\hline S35K $\quad$ F143E & Phe 142 plays a critical role in aromatic substrate binding. \\
\hline F176E & Charged residues are important at the active site entrance. \\
\hline F143A & Luminol binds to active site via electrostatic interactions in binding area. \\
\hline \multicolumn{2}{|c|}{ Proximal Region. $[102 \& 36]$} \\
\hline F179A H170A & Phe 179 is crucial for aromatic substrate binding. \\
\hline $\mathrm{F} 179 \mathrm{H}$ & His 170 tethers heme moiety in position. Prevents distal His coordination. \\
\hline F179S & His 170 maintains heme moiety in penta-coordinated state. \\
\hline \multicolumn{2}{|c|}{ Asparagine 70. $[103]$} \\
\hline N70V N70D & $\begin{array}{l}\text { Asn } 70 \text { hydrogen bonds to His } 42 \text {, mutations alter distal heme orientation. } \\
\text { Mutant protein displays increased redox potential. }\end{array}$ \\
\hline \multicolumn{2}{|c|}{ Tryptophan 117. [33 \& 104] } \\
\hline \multirow[t]{2}{*}{ W117F } & Mutants displayed increased acid stability. \\
\hline & Trp 117 is important in internal electron transfer and protein unfolding. \\
\hline \multicolumn{2}{|c|}{ Threonine 171. [37] } \\
\hline T171S & Proximal structural alteration, affects proximal pocket hydrogen bonding. \\
\hline \multicolumn{2}{|c|}{ Phenylalanine 221. [105 \& 32] } \\
\hline F221M F221W & $\begin{array}{l}\text { Mutants display decreased stability in alkaline conditions. } \\
\text { Trp introduction destabilises protein, due to unfavourable surroundings. }\end{array}$ \\
\hline
\end{tabular}

Footnote:

Mutants are grouped into active site, active site entrance, proximal heme region, aspargine 70 , tryptophan 117 and Phe 221 mutations. Mutants were expressed in a variety of hosts including E.coli, Trichoplusia ni, and Spodoptera fruigiperda cell lines. Key references only are noted for each collection of mutants; further references may be found within these. 


\begin{tabular}{|c|c|c|c|c|c|c|c|}
\hline Electrode type & $\begin{array}{c}\text { Size of } \\
\text { Electrode }\end{array}$ & Enzyme & $\begin{array}{c}\text { Method of } \\
\text { immobilisation }\end{array}$ & Mediator & $\begin{array}{c}\text { Analyte } \\
\text { measured }\end{array}$ & $\begin{array}{c}\text { Method of } \\
\text { measurement }\end{array}$ & Reference \\
\hline \multicolumn{8}{|c|}{ Soybean Peroxidase } \\
\hline Glassy carbon & $4 \mathrm{~mm}$ & SBP & Adsorption & PVA/PVP & $\mathrm{H}_{2} \mathrm{O}_{2}$ & Amperometry & {$[106]$} \\
\hline Glassy carbon & $4 \mathrm{~mm}$ & SBP & Entrapment & Sol-gel & $\mathrm{H}_{2} \mathrm{O}_{2}$ & Amperometry & {$[56]$} \\
\hline Glassy carbon & $3 \mathrm{~mm}$ & SBP & Entrapment & $\begin{array}{l}\text { Pos-EA, } \\
\text { PEGDGE }\end{array}$ & $\mathrm{H}_{2} \mathrm{O}_{2}$ & Amperometry & [59] \\
\hline $\begin{array}{c}\text { Pyrolitic } \\
\text { graphite } \\
\text { (Rotating disk) }\end{array}$ & $0.2 \mathrm{~mm}$ & SBP & Entrapment & DMPC & $\mathrm{H}_{2} \mathrm{O}_{2}$ & $\begin{array}{l}\text { Amperometry } \\
\text { /CV }\end{array}$ & [107] \\
\hline Glassy carbon & $3 \mathrm{~mm}$ & GOx/SBP & Entrapment & Pos-EA & Glucose & Amperometry & {$[58]$} \\
\hline
\end{tabular}

\begin{tabular}{|c|c|c|c|c|c|c|c|}
\hline \multicolumn{8}{|c|}{ Horseradish Peroxidase } \\
\hline Gold & - & HRP & Adsorption & DNA & $\mathrm{H}_{2} \mathrm{O}_{2}$ & Amperometry & [108] \\
\hline Glassy Carbon & $3 \mathrm{~mm}$ & HRP & Adsorption & HQ & $\mathrm{H}_{2} \mathrm{O}_{2}$ & Amperometry & {$[53]$} \\
\hline $\begin{array}{c}\text { Carbon } \\
\text { Ceramic }\end{array}$ & $6 \mathrm{~mm}$ & HRP & Covalent & Nano Au & $\mathrm{H}_{2} \mathrm{O}_{2}$ & Amperometry & [109] \\
\hline Carbon Paste & $7 \mathrm{~mm}$ & HRP & Entrapment & GP & $\begin{array}{c}\text { Biogenic } \\
\text { Amine }\end{array}$ & Amperometry & {$[110]$} \\
\hline Titanium & $0.8 \mathrm{~mm}$ & HRP & Adsorption & Thionine & $\mathrm{H}_{2} \mathrm{O}_{2}$ & Amperometry & [111] \\
\hline Platinum & $1 \mathrm{~mm}$ & HRP & Entrapment & Co(bpy) & $\mathrm{H}_{2} \mathrm{O}_{2}$ & Amperometry & [112] \\
\hline Carbon Fibre & $3 \mathrm{~mm}$ & Cho/HRP & Adsorption & Os- PVP & Choline & Amperometry & [113] \\
\hline Platinum & $7 \mathrm{~mm}$ & Cho/HRP & Adsorption & TTCA & Choline & Amperometry & 114 \\
\hline
\end{tabular}

\begin{tabular}{|c|c|c|c|c|c|c|c|}
\hline \multicolumn{2}{|l|}{ Recombinant HRP } & Adsorption & Direct & $\mathrm{H}_{2} \mathrm{O}_{2}$ & Amperometry & {$[115]$} \\
$\begin{array}{c}\text { Graphite } \\
\text { Rotating disk }\end{array}$ & $3 \mathrm{~mm}$ & $\mathrm{rHRP}$ & Adsorption & Direct & $\mathrm{H}_{2} \mathrm{O}_{2}$ & Amperometry & {$[116]$} \\
Gold & $0.3 \mathrm{~mm}$ & $\begin{array}{c}\mathrm{CytC} / \\
\mathrm{rHRP}\end{array}$ & Adsoprtion & Direct & $\begin{array}{c}\text { Superoxid } \\
\text { e Anion } \\
\text { radical }\end{array}$ & Amperometry & {$[117]$} \\
Gold & $0.15 \mathrm{~mm}$ & $\begin{array}{c}\text { L-LO / } \\
\mathrm{rHRP}\end{array}$ & Adsorption & Direct & L-lysine & Amperometry & {$[118]$} \\
\hline
\end{tabular}

Table 3: Some SBP, HRP and rHRP biosensors, and their properties, from the literature. 